\title{
THE ANALYTIC THEORY OF SYSTEMS OF PARTIAL DIFFERENTIAL EQUATIONS ${ }^{1}$
}

\section{A. ERDÉLYI}

1. Introduction. The theory of ordinary linear differential equations in the complex domain has always attracted particular attention, and topics such as singularities of the regular type and equations of the Fuchsian class belong to those well-rounded gems for which the classical theory of functions of a complex variable is so justly famous.

Almost simultaneously with the fundamental researches of Fuchs and others on ordinary differential equations, efforts were made to develop a similar theory for systems of partial differential equations. Although mathematicians of the rank of Appell, Goursat, and Picard were among those interested in the matter, at the time these efforts were doomed to failure because of the insufficient understanding of functions of several complex variables, and also of some relevant topics in multiple series, algebraic geometry, topology, and groups. Today the problem could be attacked with greater hope of success, and the present survey seeks to prepare the ground for such an attack.

2. Ordinary differential equations. It will be useful to review briefly the theory of ordinary linear differential equations

$$
\frac{d^{n_{z}}}{d x^{n}}+g_{1}(x) \frac{d^{n-1} z}{d x^{n-1}}+\cdots+g_{n}(x) z=0
$$

in the complex domain. The $g_{i}(x)$ are one-valued analytic functions of the complex variable $x$, and we shall assume that each $g_{i}(x)$ has at most a finite number of singularities. It is well known that such an equation has $n$ linearly independent solutions, and that any $n+1$ solutions are linearly dependent. If all the $g_{i}(x)$ are regular in a neighborhood of $x=a$, then $a$ is an ordinary point of the equation, and in a neighborhood of any such point the equation possesses a fundamental system of $n$ analytic solutions represented by convergent power series. A singularity of any of the $g_{i}(x)$ is a singular point of the differential equation.

An address delivered before the Stanford meeting of the Society on April 28, 1951 by invitation of the Committee to Select Hour Speakers for Far Western Sectional Meetings; received by the editors April 30, 1951.

${ }^{1}$ Prepared under Contract N6onr-244 Task Order XIV of the Office of Naval Research with the California Institute of Technology. 
Let $z(x)$ be a vector of $n$ linearly independent analytic solutions $z_{1}(x), \cdots, z_{n}(x)$. If $x$ describes a closed curve $\Gamma$ every point of which is an ordinary point, then $z(x)$ can be continued analytically along $\Gamma$, and when we return to the point of departure, $z(x)$ will have changed into $\tilde{z}(x)$. Since every component of $\tilde{z}(x)$ is a solution of the differential equation, and hence a linear combination of $z_{1}(x), \cdots, z_{n}(x)$, we have a relation $\tilde{z}(x)=A z(x)$ where $A$ is an $n \times n$ matrix of constant elements. For a given differential equation, $A$ depends on $\Gamma$; for a fixed $\Gamma$, different fundamental systems give rise to equivalent matrices, and thus $\Gamma$ determines the canonical form of $A$. The linear transformations $A$ for all possible closed curves form a group, the monodromic group of the equation, and this group is generated by the transformations corresponding to simple closed curves encircling one of the singularities.

Let $x=a$ be a singular point. If the canonical form of the corresponding matrix $A$ is diagonal,

$$
\left[\begin{array}{lll}
e^{2 i \pi \rho_{1}} & & \\
& \ddots & \\
& & \\
& & e^{2 \pi i \rho_{n}}
\end{array}\right],
$$

then there is a fundamental system of the form $z_{j}=(x-a)^{\rho} y_{j}(x)$ where the $y_{j}$ are holomorphic in a deleted neighborhood of $x=a$, and hence can be represented by Laurent series. If the canonical form of $A$ is not diagonal, logarithmic solutions occur in the fundamental system but otherwise the situation is unchanged. In case the Laurent series have only a finite number of terms with negative exponents, the $\rho_{j}$ can be adjusted so that the $y_{j}$ are all analytic at $x=a$. A point at which this is possible is a regular singular point, and equations possessing a finite number of regular singularities, and no other singularities, are said to be of the Fuchsian class.

A linear differential equation determines its monodromic group; and Riemann's investigations of hypergeometric functions show that at any rate Fuchsian equations are essentially determined by their monodromic group. More precisely, for an equation of the $n$th order with $m$ regular singularities, the singularities can be prescribed arbitrarily. If $m n \leqq 2(n+1)$, the monodromic group is subject to $\left(n^{2}-1\right)$ $-n(n-1) m / 2$ conditions; and any group satisfying these conditions can be prescribed and then determines the equation completely. If $m n>2(n+1)$ any (consistent) monodromic group can be prescribed and determines the differential equation up to $n(n-1) m / 2-\left(n^{2}-1\right)$ arbitrary constants (the accessory parameters). 
3. Systems of partial differential equations. The extension of all these ideas to several complex variables was envisaged as early as 1880 when Appell [1 $]^{2}$ introduced certain series in two variables $x$ and $y$ which are analogous to the hypergeometric series $F(\alpha, \beta ; \gamma ; x)$. Appell's series satisfy a system of partial differential equations of the second order of the form

$$
\begin{aligned}
& r=a s+b p+c q+e z, \\
& t=f s+g p+h q+k z
\end{aligned}
$$

where $z$ is the unknown function, $p=z_{x}, q=z_{y}, r=z_{x x}, s=z_{x y}, t=z_{y y}$, subscripts denote partial differentiation, and $a, b, c, \cdots, k$ are given (in this case rational, more generally analytic) functions of the independent variables $x$ and $y$. Investigations by Bouquet, Méray, and Mayer give information about the number of linearly independent solutions of (2), and a certain amount of information about the singularities; but it is significant that in no case is complete information available. The best known system is that belonging to Appell's function $F_{1}$, that is, the system

$$
\begin{aligned}
x(1-x) r+y(1-x) s+\{\gamma-(\alpha+\beta+1) x\} p & \\
& -\beta y q-\alpha \beta z=0, \\
y(1-y) t+x(1-y) s+\left\{\gamma-\left(\alpha+\beta^{\prime}+1\right) y\right\} q & -\beta^{\prime} x p-\alpha \beta^{\prime} z=0
\end{aligned}
$$

( $\alpha, \beta, \beta^{\prime}, \gamma$ constants), and although 10 solutions of (3) were known as early as 1893 (LeVavasseur) [17], it turns out that these 10 solutions are insufficient to provide fundamental systems for the neighborhood of all singular points. For this another 15 solutions are necessary and these were obtained only recently (Erdélyi [4]) by rather ad hoc methods, not from the general theory.

Much of this survey will be restricted to a discussion of systems of the form (2). The most significant exception from this rule is the next section which contains a brief account of the modern investigations into the existence theory of analytic solutions of systems of partial differential equations. Here we can handle a much more general situation.

4. Existence theorems. Given a system of partial differential equations in any number of variables and unknown functions, we may ask whether the system is consistent, whether it has analytic solutions, and also we may ask for the number of arbitrary constants

${ }^{2}$ Numbers in brackets refer to the references at the end of the paper. 
(or arbitrary analytic functions) contained in the general (analytic) solution of the system.

Such questions were first investigated by Cauchy and Sonja Kovalewska [16] and it was found that a formal power-series solution is no guarantee for the existence of an analytic solution. A more detailed investigation was commenced by Méray [20] and Riquier $[23 ; 24]$, and continued by Janet $[13 ; 14 ; 15]$. With a view of ordering derivatives integers, called "cotes," were attached to derivatives, and majorization was used to prove the existence of analytic solutions. Riquier actually succeeded in proving the existence of analytic solutions for so-called orthonomic systems, and even for more general systems in case the defining functions and initial determinations satisfy certain inequalities.

A more modern version of the theory is due to J. M. Thomas $[27 ; 28 ; 29 ; 30 ; 31 ; 32]$. Eventually Thomas was able to dispense with "cotes," and develop an algebraic procedure which resulted in existence theorems wider than Riquier's.

Let $x_{1}, \cdots, x_{n}$ be the independent variables, $u_{1}, \cdots, u_{r}$ the unknown functions.

$$
D=\frac{\partial^{i_{1}+\cdots+i_{n}} u_{j}}{\partial x_{1}^{i_{1}} \cdots \partial x_{n}^{i_{n}}}
$$

is a typical derivative. We shall associate it with the monomial

$$
m=x_{1}^{i_{1}} \cdots x_{n}^{i_{n}} u_{j},
$$

and write $D \sim m$. We consider a system $S$ consisting of a finite number of partial differential equations of finite order, each solved explicitly for one of the derivatives. Thus $S$ consists of equations of the form

$$
D_{i}^{\prime}=f_{i}\left(x, D^{\prime \prime}\right), \quad i=1, \cdots, k,
$$

each of which involves only a finite number of derivatives $D^{\prime \prime}$. A set of constants for the $x$ and the $D$ which substituted in (4) satisfy each equation is called a numerical determination, and the values of the $x$ in a numerical determination are the initial values. It is assumed that each $f\left(x, D^{\prime \prime}\right)$ is an analytic function of the $x$ and the $D$, holomorphic in some neighborhood of the numerical determination.

Let $D^{\prime}$ be a derivative occurring on the left-hand side of an equation (4), and $D^{\prime \prime}$ any of the derivatives occurring on the righthand side of the same equation: $D^{\prime} \sim m^{\prime}, D^{\prime \prime} \sim m^{\prime \prime}$. If there are positive constants $x, u$ satisfying all inequalities $m^{\prime}>m^{\prime \prime}, x_{i}>1$, Thomas 
says that the first inequality system is consistent, and calls the system $S$ admissible. The derivatives can be ordered by the adopted solution of the first inequality system. An admissible system can be prolonged by differentiating each equation with respect to the $x$ in every possible way, taking account of the dependence of the $D$ on the $x_{j}$; the infinite system so obtained is the prolongation of $S$. An initial determination of the prolongation gives initial values of all $D$, and thus a formal power series solution, known as the tentative solution; the number of arbitrary $D$ in the initial determination gives information about the number of arbitrary constants in the general solution of $S$.

Next a second inequality system is obtained whose consistency means, roughly, that a certain principal part $S^{*}$ of the prolongation can be ordered. An admissible system whose second inequality system is consistent is an orthonomic system. In general, $S^{*}$ can be decomposed, and if every component of $S^{*}$ has a consistent second inequality system, then $S$ is said to be orderly. The numerical determination is part of the orderly system. The expansions of a tentative solution of an orderly system converge in a neighborhood of the initial values; whether or not the tentative solution actually satisfies the system $S$ depends on the particular initial determination chosen. However, if a series of further conditions is satisfied, the system is passive, and it can be proved that a passive orderly system has a unique analytic solution corresponding to each arbitrarily given (relevant) initial determination.

Clearly the theory thus sketched is restricted to ordinary points of the system, that is, to points in the neighborhood of which the general (analytic) solution is regular.

5. Total differential equations. We now take a system of linear partial differential equations in two independent variables $x$ and $y$ and one unknown function $z$, and use the notation introduced in $\S 3$ for partial derivatives. First we shall show that such a system can be converted into a system of total differential equations in two independent variables (see Appell and Kampé de Fériet [2], Chapter 3, and the literature quoted there).

We take a system of the form (2) and assume at first that $1-a f$ does not vanish identically. Differentiate the first equation with respect to $y$, and the second with respect to $x$. Since $r_{y}=s_{x}$ and $t_{x}=s_{y}$, we obtain two equations for $s_{x}$ and $s_{y}$, and since $1-a f$ does not vanish identically, these two equations can be solved in the form

$$
s_{x}=\alpha s+\beta p+\gamma q+\delta z, \quad s_{y}=\epsilon s+\eta p+\theta q+\zeta z .
$$


In this case we obtain the following system in the four unknown functions $z, p, q, s$

$$
\begin{aligned}
d z & =p d x+q d y, \\
d p & =(a s+b p+c q+e z) d x+s d y, \\
d q & =s d x+(f s+g p+h q+k z) d y, \\
d s & =(\alpha s+\beta p+\gamma q+\delta z) d x+(\epsilon s+\eta p+\theta q+\zeta z) d y .
\end{aligned}
$$

On the other hand, if $1-a f$ vanishes identically, then all third-order derivatives can be eliminated from the differentiated equations (2), and the result is an equation of the form

$$
\alpha r+\beta s+\gamma t=\delta p+\epsilon q+\eta z,
$$

so that in this case (2) is equivalent to a system of three partial differential equations of the second order. If

$$
\left|\begin{array}{rrr}
1 & -a & 0 \\
0 & -f & 1 \\
\alpha & \beta & \gamma
\end{array}\right|=\left|\begin{array}{ccc}
1 & -a & 0 \\
0 & -f & 1 \\
a g & a\left(h+f_{x}\right)+f\left(b+a_{y}\right) & c f
\end{array}\right|
$$

does not vanish identically, the system (2), (6) can be replaced by

$$
r=A z+B p+C q, \quad s=D z+E p+F q, \quad t=G z+H p+K q,
$$

and we have now a system of total differential equations in the three unknown functions $z, p, q$ of $x$ and $y$,

$$
\begin{aligned}
d z & =p d x+q d y, \\
d p & =(A z+B p+C q) d x+(D z+E p+F q) d y, \\
d q & =(D z+E p+F q) d x+(G z+H p+K q) d y .
\end{aligned}
$$

If the determinant (7) vanishes identically, a further reduction takes place, and one obtains a system in less than three unknown functions.

In any event, a system of linear partial differential equations can be converted into a system $S$ of total linear differential equations in two independent variables,

$$
d z_{k}=\sum_{j}\left[f_{k j}(x, y) d x+g_{k j}(x, y) d y\right] z_{j}, \quad k, j=1, \cdots, n .
$$

Such a system must satisfy the compatibility conditions

$$
\frac{\partial}{\partial y}\left(\sum_{j} f_{i j} z_{i}\right)=\frac{\partial}{\partial x}\left(\sum_{i} g_{i j} z_{j}\right)
$$


or

$$
\sum_{j}\left(f_{i j} g_{j k}-g_{i j} f_{j k}\right)=\frac{\partial g_{i k}}{\partial x}-\frac{\partial f_{i k}}{\partial y}, \quad i, j, k=1, \cdots, n .
$$

If these conditions are satisfied, it is known that (10) has a fundamental system of $n$ solutions $z_{k}^{m}, k, m=1, \cdots, n$, which are analytic at every point at which all the $f_{k j}$ and $g_{k j}$ are analytic (ordinary point).

6. Singularities. For the further discussion let us assume that the $f$ and $g$ in (10) are rational functions of $x$ and $y$ which satisfy (11). From the foregoing it is clear that singularities of the differential equations are caused by the denominators of the $f$ or $g$. Let $\phi(x, y)$ be an irreducible factor of one of the denominators. The algebraic curve $\phi(x, y)=0$ is a singular curve of the system (7).

Every closed curve $\Gamma$ in the four-dimensional $x, y$ space which avoids all singular curves of (10) corresponds to a transformation of our fundamental system, and the totality of transformations corresponding to all such $\Gamma$ form the monodromic group of the equation. It appears that not much progress has been made in the investigation of the monodromic group.

Let the irreducible algebraic curve $\phi(x, y)=0$, or $C$, be a singular curve of $S$. A point $(a, b)$ of $C$ is called a nonsingular point of $C$ if either $\phi_{x}(a, b) \neq 0$ or $\phi_{y}(a, b) \neq 0$; and it is called a general point of $C$ if it is nonsingular and if $C$ is the only singular curve of the system which passes through that point. If there is a fundamental system of solutions which can be represented in the canonical form

$$
z_{k}^{m}=\phi^{\rho_{m}} p_{k}^{m}(z-a, y-b)
$$

(or in special cases in a similar form containing terms in $\log \phi$ ), where the $p(x-a, y-b)$ are power series convergent in some neighborhood of $(a, b)$; and if such a representation holds in the neighborhood of each general point $(a, b)$ of $C$, with the $\rho_{m}$ independent of $k, a, b, x, y$, then $\phi=0$ may be called a singular curve of the regular type.

A point $(a, b)$ will be called a general intersection of the two irreducible singular curves $\phi(x, y)=0, \psi(x, y)=0$, if it is a nonsingular point both of $\phi=0$ and $\psi=0$, if no other singular curve passes through it, and if the Jacobian $\partial(\phi, \psi) / \partial(x, y) \neq 0$ at $(a, b)$. In the neighborhood of a general intersection of two singular curves $\phi=0$, $\psi=0$ of the regular type, a canonical fundamental system is of the form 


$$
z_{k}^{m}=\phi^{\rho_{m}} \psi^{\sigma_{m}} p_{k}^{m}(x-a, y-b)
$$

(or logarithmic solutions). It appears that at present the general theory cannot handle a point which is an intersection of three or more singular curves, or at which two curves are in contact $(\partial(\phi, \psi) / \partial(x, y)=0)$, or which is a singular point of a singular curve $\left(\phi_{x}=\phi_{y}=0\right)$. As J. F. Ritt remarks in the introduction to his Differential equations from the algebraic standpoint [25], canonical forms, which are usually assumed in dealing with systems of differential equations, are inadequate for the representation of general systems since in the process of reduction to canonical forms one encounters so many exceptions, possibilities of degeneration, and possibilities of the occurrence of extraneous factors as to make the general theory somewhat less fruitful than the investigation of special systems. The study of particular examples suggests an entirely novel occurrence in the analytic theory of differential equations: there does not seem to be any fundamental system which is canonical in the entire neighborhood of such a point. In the case of a general intersection of three singular curves the examples indicate the existence of three distinct canonical systems.

Returning to the system of partial differential equations (2), we have to distinguish two cases. If $1-a f$ does not vanish identically, then the system can be transformed into a system of total differential equations of the form (5), and if the compatibility conditions are satisfied for this system, (2) has four linearly independent solutions. The singular curves of (2) are, in this case, the singular curves of $a, \cdots, k$ and the curve or curves determined by $1-a f=0$.

If $1-a f$ vanishes identically, but the determinant (7) does not vanish identically, then the system (2) can be transformed into a system of total differential equations of the form (9), and if the compatibility conditions for this system are satisfied, (2) has three linearly independent solutions. The singular curves of (2) are, in this case, the singular curves of $a, \cdots, k$ and the zero curve or curves of the determinant (7).

There is no need to give a discussion of further special cases. It is clear that in any given case an equivalent system of total differential equations can be derived, the compatibility conditions can be checked, and the prospective singular curves of the system can be obtained. The curves so obtained may be real or apparent singular curves of the system (2), and if they are in reality singular curves of the system they may be of the regular or irregular type. It appears that no general criterion is known for finding out which of these possi- 
bilities is present in any given case. Furthermore, even if it is known that certain singular curves are regular singular curves of the system, the general theory furnishes the form of the canonical fundamental system only for a general point of regular singular curve, or for a general intersection of two singular curves. All points of a more complicated nature require a special investigation, and except for a very few examples there is little hint as to the nature of this investigation.

7. Examples. At this stage it seems worth while to consider a few examples and see how the general theory applies, and wherein it fails to give the required answers. We shall choose hypergeometric systems of partial differential equations, because about these a considerable amount of information is available.

The system (3) is satisfied by Appell's series

$$
F_{1}\left(\alpha, \beta, \beta^{\prime}, \gamma ; x, y\right)=\sum \sum \frac{(\alpha)_{\lambda+\mu}(\beta)_{\lambda}\left(\beta^{\prime}\right)_{\mu}}{(\gamma)_{\lambda+\mu} \lambda ! \mu !} x^{\lambda} y^{\mu}
$$

and is thus compatible. It can be written in the form (2), 1-af of that form vanishes identically, and apart from an irrelevant factor the determinant $(7)$ is $x-y$, so that the system has three linearly independent solutions. Clearly the singular curves of the system are

$$
x=0, \quad x=1, \quad x=\infty, \quad y=0, \quad y=1, \quad y=\infty, \quad x-y=0,
$$

and it is easy to see that they all are of the regular type. Each of the six singular points $(0,1),(0, \infty),(1,0),(1, \infty),(\infty, 0),(\infty, 1)$ is a general intersection of two regular singular curves. In the neighborhood of each of these points $(a, b)$ there is a fundamental system of three solutions of the canonical form

$$
(x-a)^{\rho}(y-b)^{\sigma} p(x-a, y-b),
$$

and these solutions have been known for a long time.

The remaining three singular points, $(0,0),(1,1)$, and $(\infty, \infty)$, are of a very different nature. At each of these singular points $(a, a)$ three singular curves intersect. There is indeed one solution of the form

$$
(x-a)^{\rho}(y-a)^{\sigma} p(x-a, y-a)
$$

for each of these singular points, but no fundamental system of three linearly independent solutions of the canonical form is available. Several investigations were carried out in order to determine an appropriate form of the fundamental system. No system can be found which is canonical for every (sufficiently small) closed $\Gamma$ in the neighborhood of $(a, a)$ and for this reason Horn $[10 ; 11]$ has proposed to in- 
vestigate the behavior of solutions as $(x, y)$ approaches $(a, a)$ along a straight line rather than encircles $(a, a)$. Actually it is not really necessary to exclude closed curves $\Gamma$ encircling $(a, a)$. It turns out (Erdélyi [4]) that the entire neighborhood of the singular point $(a, a)$ must be broken up into three parts, according as to which of the three quantities $|x-a|,|y-a|,|x-y|$ is the smallest. For a closed curve $\Gamma$ which remains entirely within one of the three "conical" neighborhoods, there is a canonical fundamental system: when proceeding from one conical neighborhood to another, one must change from one canonical system to another. To begin with, one obtains six forms of canonical systems which are indicated below together with the supplementary conditions defining that part of the neighborhood of $(a, a)$ in which they are valid.

$$
\begin{array}{ll}
(x-a)^{\rho}(y-a)^{\sigma} p\left(\frac{x-a}{y-a}, y-a\right), & \left|\frac{x-a}{y-a}\right|<1, \\
(x-a)^{\rho}(x-y)^{\sigma} p\left(\frac{x-a}{x-y}, x-y\right), & \left|\frac{x-a}{x-y}\right|<1, \\
(x-a)^{\rho}(y-a)^{\sigma} p\left(x-a, \frac{y-a}{x-a}\right), & \left|\frac{y-a}{x-a}\right|<1, \\
(x-y)^{\rho}(y-a)^{\sigma} p\left(x-y, \frac{y-a}{x-y}\right), & \left|\frac{y-a}{x-y}\right|<1, \\
(x-a)^{\rho}(x-y)^{\sigma} p\left(x-a, \frac{x-y}{x-a}\right), & \left|\frac{x-y}{x-a}\right|<1, \\
(y-a)^{\rho}(x-y)^{\sigma} p\left(y-a, \frac{x-y}{y-a}\right), & \left|\frac{x-y}{y-a}\right|<1 .
\end{array}
$$

Clearly, (12) and (13) are equivalent in that conical part of the neighborhood of $(a, a)$ in which $|x-a|<\min (|y-a|,|x-y|)$. Similarly, (14) and (15), and also (16) and (17), coincide in one or the other of the conical neighborhoods. It should be noted, however, that there is a two-dimensional surface along which none of the six canonical systems is valid.

This example shows clearly how to construct canonical fundamental systems for the various conical parts of the neighborhood of a general intersection of three singular curves of the regular type, but in general it is rather difficult to determine the linear transformations which transform any one of these canonical systems into any other one. 
As another example we choose the system of partial differential equations

$$
\begin{aligned}
& x(x+1) r-y s-y^{2} t+\left\{\left(\alpha+\beta^{\prime}+1\right) x+1-\beta\right\} p \\
&+\left(\beta^{\prime}-\alpha-1\right) y q+\alpha \beta^{\prime} z=0 \\
&-x^{2} r-x s+y(y+1) t+(\beta-\alpha-1) x p \\
&+\left\{(\alpha+\beta+1) y+1-\beta^{\prime}\right\} q+\alpha \beta z=0
\end{aligned}
$$

which is satisfied by Horn's series

$$
G_{1}\left(\alpha, \beta, \beta^{\prime} ; x, y\right)=\sum \sum(\alpha)_{\lambda+\mu}(\beta)_{\mu-\lambda}\left(\beta^{\prime}\right)_{\lambda-\mu} \frac{x^{\lambda}}{\lambda !} \frac{y^{\mu}}{\mu !}
$$

and hence is consistent. This system is not of the form (2) but it can easily be transformed into that form. An investigation shows that the system has three linearly independent solutions and that its singular curves are $x=0, x=\infty, y=0, y=\infty, x+y+1=0,4 x y-1=0$. Each of the three points $(0,0),(0,-1),(-1,0)$ is a general intersection of two regular singularities, and a canonical fundamental system for each of these points can be obtained in the form predicted by the general theory. On the other hand, at the point $(-1 / 2,-1 / 2)$ the two singular curves $x+y+1=0$ and $4 x y-1=0$ are in contact, and the general theory does not give any information there. The integration of the system by contour integrals suggests the transformation

$$
\begin{aligned}
\xi & =\frac{1}{2 x}\left[1+2 x+(1-4 x y)^{1 / 2}\right], \\
\eta & =\frac{1}{2 x}\left[1+2 x-(1-4 x y)^{1 / 2}\right], \\
\zeta & =(1+x+y)^{\alpha} z
\end{aligned}
$$

and this transformation carries the two singular curves which have a common tangent at $x=y=-1 / 2$ into a general intersection of the three singular lines $\xi=0, \eta=0, \xi-\eta=0$ of the system of partial differential equations satisfied by $\zeta$ as a function of $\xi$ and $\eta$; and we already know how to deal with such an intersection.

There are other examples showing how to "reduce," by a transformation, a singular point, for instance a cusp, of a singular curve. Generally speaking, given any type of singular point of a system of partial differential equations, it is usually easy to reduce this singular point locally, that is, to find a transformation which will map this singular point into a finite number of singular points each of which is a general intersection of two singular curves. There is no guarantee, 
however, that this can be performed simultaneously for all singular points. Given a finite number of algebraic curves, Professor H. Busemann informs me that in general there is no birational transformation of the $x, y$ plane into itself which will reduce (in the fashion described above) all singular points of these curves, and all their intersections; and although it is known from algebraic geometry that the singular points of any algebraic curve can be reduced to ordinary double points by a birational transformation defined on the curve only (Riemann transformation), such transformations do not appear to be very useful in connection with differential equations.

8. Riemann's problem. We shall say that an analytic function of $x$ is of canonical form, and has exponent $\rho$, at $x=a$ if it is of the form $(x-a)^{\circ} p(x-a)$ where $p$ is a power series convergent in some neighborhood of $a$. When $a=\infty$, the appropriate form is $x^{-\rho} p(1 / x)$. Similarly, an analytic function of $x$ and $y$ is of canonical form, and has exponent $\rho$, at the singular curve $\phi(x, y)=0$ if in the neighborhood of any general point $(a, b)$ of that curve it is of the form $\phi^{p} p(x-a, y-b)$.

Riemann has shown that the differential equation

$$
x(1-x) \frac{d^{2} z}{d x^{2}}+[\gamma-(\alpha+\beta+1) x] \frac{d z}{d x}-\alpha \beta z=0
$$

can be identified as the differential equation satisfied by the family $P$ of analytic functions of $x$ described as follows.

(i) $\alpha, \beta, \gamma$ are given constants; $\alpha-\beta, \gamma, \gamma-\alpha-\beta$ are not integers.

(ii) Each function of $P$ is holomorphic everywhere except possibly for $x=0,1, \infty$.

(iii) Any three functions of $P$ are linearly dependent.

(iv) At $x=0$ there are two functions of $P$ which are in canonical form with exponents 0 and $1-\gamma$, similarly at $x=1$ with exponents 0 and $\gamma-\alpha-\beta$, and at $x=\infty$ with exponents $\alpha$ and $\beta$.

Picard [21] attempted to characterize in a similar manner the system (3) of partial differential equations satisfied by Appell's function $F_{1}$ as the system satisfied by all members of a family $P$ of analytic functions of $x$ and $y$ with the following properties.

(i) $\alpha, \beta, \beta^{\prime}, \gamma$ are given constants, $\alpha-\beta, \alpha-\beta^{\prime}, \beta+\beta^{\prime}, \gamma-\beta, \gamma-\beta^{\prime}$, $\gamma-\alpha-\beta, \gamma-\alpha-\beta^{\prime}$ are not integers.

(ii) Each function of $P$ is holomorphic, except when $x=0,1, \infty$, or $y=0,1, \infty$, or $x=y$.

(iii) Any four functions are linearly dependent

(iv) At each of the singular curves there are three linearly inde- 
pendent functions of $P$ with the exponents given in the table.

Singular curve

$$
\begin{aligned}
& x=0 \\
& x=1 \\
& x=\infty \\
& y=0 \\
& y=1 \\
& y=\infty \\
& x=y
\end{aligned}
$$

Exponents

$$
\begin{aligned}
& 0,0,1+\beta^{\prime}-\gamma \\
& 0,0, \gamma-\alpha-\beta \\
& \alpha, \beta, \beta \\
& 0,0,1+\beta-\gamma \\
& 0,0, \gamma-\alpha-\beta^{\prime} \\
& \alpha, \beta^{\prime}, \beta^{\prime} \\
& 0,0,1-\beta-\beta^{\prime}
\end{aligned}
$$

If $z_{1}, z_{2}, z_{3}$ are any three linearly independent functions of $P$, then any function of $P$ satisfies the three partial differential equations

$$
\left|\begin{array}{llll}
r & p & q & z \\
r_{1} & p_{1} & q_{1} & z_{1} \\
r_{2} & p_{2} & q_{2} & z_{2} \\
r_{3} & p_{3} & q_{3} & z_{3}
\end{array}\right|=0,\left|\begin{array}{llll}
s & p & q & z \\
s_{1} & p_{1} & q_{1} & z_{1} \\
s_{2} & p_{2} & q_{2} & z_{2} \\
s_{3} & p_{3} & q_{3} & z_{3}
\end{array}\right|=0,\left|\begin{array}{llll}
t & p & q & z \\
t_{1} & p_{1} & q_{1} & z_{1} \\
t_{2} & p_{2} & q_{2} & z_{2} \\
t_{3} & p_{3} & q_{3} & z_{3}
\end{array}\right|=0 .
$$

Arguing on the exponents, Picard could show that these equations are of the same form as those satisfied by $F_{1}$, but he could not identify all constants, except by deriving the ordinary differential equation (of the third order) satisfied by any function of $P$ when $y$ is fixed and $x$ varies (or vice versa), and using the theory of Fuchsian equations, in this particular case investigations by Pochhammer [22].

A similar problem relating to Appell's series $F_{3}$, whose system of partial differential equations has four linearly independent solutions, was discussed by Goursat [5]. The list of singular curves and exponents follows:

$$
\begin{array}{ll}
\text { Singular curve } & \multicolumn{1}{c}{\text { Exponents }} \\
x=0 & 0,0,1+\alpha^{\prime}-\gamma, 1+\beta^{\prime}-\gamma \\
x=1 & 0,0,0, \gamma-\alpha-\beta \\
x=\infty & \alpha, \alpha, \beta, \beta \\
y=0 & 0,0,1+\alpha-\gamma, 1+\beta-\gamma \\
y=1 & 0,0,0, \gamma-\alpha^{\prime}-\beta^{\prime} \\
y=\infty & \alpha^{\prime}, \alpha^{\prime}, \beta^{\prime}, \beta^{\prime} \\
x+y-x y=0 & 0,0,0,1+\gamma-\alpha-\alpha^{\prime}-\beta-\beta^{\prime}
\end{array}
$$

Riemann's investigation of the hypergeometric differential equa- 
tion leads to the Fuchsian theory of linear differential equations with regular singularities. A corresponding theory of systems of partial differential equations is not feasible at present because of the lack of convenient criteria for singular curves of the regular type.

\section{REFERENCES}

1. P. Appell, Sur les séries hypergéométriques de deux variables et sur les équations différentielles linéaires aux dérivées partielles, C. R. Acad. Sci. Paris vol. 90 (1880) pp. 296-298 and 731-735.

2. P. Appell and J. Kampé de Fériet, Fonctions hypergéométriques et hypersphériques. Polynomes d'Hermite, Paris, 1926.

3. M. Bouquet, Sur l'intégration d'un système d'équations différentielles totales simultanées du premier ordre, Bull. Sci. Math. vol. 3 (1872) pp. 265-274.

4. A. Erdélyi, Hypergeometric functions of two variables, Acta Math. vol. 83 (1950) pp. 131-164.

5. É. Goursat, Extension du problème de Riemann à des fonctions hypergéométriques de deux variables, C. R. Acad. Sci. Paris vol. 95 (1882) pp. 903-906, 1044-1047.

6. J. Horn, Zur Integration der Systeme totaler linearer Differentialgleichungen mit zwei unabhängigen Veränderlichen, Math. Ann. vol. 42 (1893) pp. 215-252.

7. - - Hypergeometrische Funktionen zweier Vernäderlichen, Math. Ann. vol. 105 (1931) pp. 381-407.

8. - - Hypergeometrische Funktionen zweier Veränderlichen, Math. Ann. vol. 111 (1935) pp. 638-677.

9. - - Hypergeometrische Funktionen zweier Veränderlichen, Math. Ann. vol. 113 (1936) pp. 242-291.

10. - Über eine hypergeometrische Funktion zweier Veränderlichen, Monatshef te für Mathematik vol. 47 (1938) pp. 186-194.

11. - Hypergeometrische Funktionen zweier Veränderlichen im Schnittpunkt dreier Singularitäten, Math. Ann. vol. 115 (1938) pp. 435-455.

12. - Über eine hypergeometrische Funktion zweier Veränderlichen, Monatshefte für Mathematik vol. 47 (1939) pp. 359-379.

13. M. Janet, Les systèmes d'équations aux dérivées partielles, Journal de Mathématiques vol. 3 (1920) pp. 65-151.

14. —- Les systèmes d'équations aux dérivées partielles, Mémorial des Sciences Mathématiques, no. 21, Paris, 1927.

15. - Leşons sur les systèmes d'équations aux dérivées partielles, Paris, 1929.

16. S. Kowalevsky, Zur Theorie der partiellen Differentialgleichungen, Journal für Mathematik vol. 80 (1875) pp. 1-32.

17. R. LeVavasseur, Sur le system d'équations aux dérivées partielles simultanées auxquelles satisfait la série hypergéométrique à deux variables $F_{1}\left(\alpha, \beta, \beta^{\prime}, \gamma, x, y\right)$, Thèse, 1893.

18. A. Mayer, Ueber unbeschränkt integrable Systeme von linearen totalen Differentialgleichungen und die simultane Integration linearer partieller Differentialgleichungen, Math. Ann. vol. 5 (1872) pp. 448-470.

19. P. Méray, Nouveau précis d'analyse infinitésimale, 1872, p. 143.

20. - Démonstration générale de l'existence des intégrales des équations aux dérivées partielles, Journal de Mathématiques (3) vol. 6 (1880) pp. 235-266.

21. É. Picard, Sur une extension aux fonctions de deux variables du problème de Riemann relatif aux fonctions hypergéométriques, Ann. École Norm. (2) vol. 10 (1881) 
pp. 305-322 (cf. also C. R. Acad. Sci. Paris vol. 90 (1880) pp. 1119-1121, 1267-1269).

22. L. Pochhammer, Über hypergeometrische Funktionen höherer Ordnungen, J. Reine Angew. Math. vol. 71 (1870) pp. 316-352.

23. C. Riquier, Les systèmes d'équations aux dérivées partielles, Paris, 1910.

24. - La méthode des fonctions majorantes et les systèmes d'équations aux dérivées partielles, Mémorial des Sciences Mathématiques, no. 32, Paris, 1928.

25. J. F. Ritt, Differential equations from the algebraic standpoint, Amer. Math. Soc. Colloquium Publications, vol. 14, New York, 1932.

26. - Differential algebra, Amer. Math. Soc. Colloquium Publications, vol. 33, New York, 1950.

27. J. M. Thomas, Riquier's existence theorems, Ann. of Math. (2) vol. 30 (1929) pp. $285-310$.

28. - The condition for an orthonomic differential system, Trans. Amer. Math. Soc. vol. 34, (1932) pp. 332-338.

29. ——, Riquier's existence theorems, Ann. of Math. (2) vol. 35 (1934) pp. 306311.

30. - Differential systems, Amer. Math. Soc. Colloquium Publications, vol. 21, New York, 1937.

31. ——, Orderly differential systems, Duke Math. J. vol. 7 (1940) pp. 249-290.

32. —_ Positive solutions of binomial inequalities, Duke Math. J. vol. 7 (1940) pp. 291-297.

California Institute of Technology 